\title{
Optimization parameters effects on electrical conductivity of 3D printed circuits fabricated by direct ink writing method using functionalized multiwalled carbon nanotubes and polyvinyl alcohol conductive ink
}

\author{
Syed Riyaz Ahammed* and Ayyappan Susila Praveen \\ Department of Mechanical Engineering, Vel Tech Rangarajan Dr. Sagunthala R\&D Institute of Science and Technology, Avadi, \\ Chennai, Tamil Nadu, India
}

Received: 21 April 2021 / Accepted: 4 June 2021

\begin{abstract}
Fabrication of electronic circuits and the effects of optimization parameters on electrical conductivity of the printed circuits fabricated by direct ink writing method (D.I.W); one of the novel methods in 3D printing technologies is discussed in this work. This paper focuses on fabrication of electronic circuits using F-MWCNT/PVA conductive ink and analyses the effect of input printing process parameters namely nozzle diameter, extrusion pressure, printing speed on evaluating the electrical conductivity. Box-Behnken approach is followed to generate the levels of experiments and the performance of developed model is assessed using ANOVA. Response surface method is incorporated to find the influencing parameters on electrical conductivity response. Two-point probe measurement method is performed to analyse the output response of the printed electronic circuits. Optimized printing parameters such as nozzle diameter of $0.8 \mathrm{~mm}$, extrusion pressure of $0.1 \mathrm{MPa}$ and printing speed of $4 \mathrm{~mm} / \mathrm{sec}$ are found to be the best the for printing electronic circuits with high electrical conductivity.
\end{abstract}

Keywords: Direct ink writing / MWCNT/PVA conductive ink / Box-Behnken design / ANOVA / response surface methodology

\section{Introduction}

Direct ink writing (D.I.W) method, one of the types of 3D printing technology has attracted researchers in recent years and has been recognised as an advanced fabrication technology because of the wide scope of applications in manufacturing flexible, wearable electronic circuits and components [1-5] e.g., printing flexible circuits [6,7], wearable electronics $[8,9]$, super capacitors [10,11], batteries $[12,13]$, strain sensor [14,15], energy harvesting devices $[16,17]$, touch screens [18,19], bio sensors [20-22]. This D.I.W technology advances in the fabrication of complex electronic circuits by adopting pneumatic extrusion process [23-25] by formulating ink with controlled rheology [26-28]. D.I. W is a one step 3D printing method; overcomes the fused deposition modelling (FDM) by avoiding the process of formulation of suitable filament from the conductive ink and promotes the scope to analyse advanced composite materials and its processing with high perfor-

\footnotetext{
* e-mail: syedriyaz@veltech.edu.in
}

mance [29]. DIW process print circuits without template supports and enables the design process to be easier and simplified by eliminating the need for tooling, masks and reducing the post processing. This printing process trends to be shortening of manufacturing cylces and production cost compared to other manufacturing methods [30].

D.I.W process starts with the 3D modelling of required prototype; sliced in to $2 \mathrm{D}$ digital models by supporting software that is translated in to the code usable by $3 \mathrm{D}$ printer; printed layer by layer [31]. Printability of inks is very essential in D.I.W as they determine the performance of fabricated parts. In printed electronics, conductive inks can impart flexibility [4-8,11,32], mechanical robustness and electrical conductivity [15-19,33]. Many researchers have fabricated electronic circuits by D.I.W method with printable inks which are composites of nano fillers like CNT, graphene, carbon black, silver inks and polymers like polyvinyl alcohol (PVA), polydimethylsiloxane (PDMS), polyvinylidene fluoride (PVDF), polyaniline (PANI) $[11,14,15,18,22,34,35]$.

In the last few years, researchers have fabricated electronic circuits and components following the D.I.W 
method using novel conductive composite inks and reported the performance of circuits in reference to electrical conductivity. Abas et al. have discussed the fabrication of a very low cost flexible electronic circuit using carbon paste by adopting D.I.W method and observed the conductivity of printed circuits to be $4.26 \times 10^{1} \mathrm{~S} / \mathrm{m}$ [4]. Luo et al. demonstrated the feasibility of fabricating CNT/ (PDMS) circuits by D.I.W method with a conductivity of $1.80 \times 10^{2} \mathrm{~S} / \mathrm{m}$ [8]. Electrical conductivity of the graphene based circuits fabricated by Shi et al. was observed to be $6 \times 10^{2} \mathrm{~S} / \mathrm{m}$ [14]. Hu et al. printed silver coated Polysterene/PDMS based strain circuits with a high conductivity of $6 \times 10^{4} \mathrm{~S} / \mathrm{m}$ and proposed that electrical conductivity of printed circuits can be enhanced by decorating conductive inks with silver, gold nano particles [16]. Capacitive touch pad printed by Nitheesh et al. using silver nanowire ink by D.I.W method presented the conductivity to be $8.3 \times 10^{2} \mathrm{~S} / \mathrm{m}$ [18].

Although several articles report the electrical conductivity performance of the printed circuits fabricated by D.I.W method using different conductive inks, optimization parameters that influence the electrical conductivity response of the printed circuits is very essential to be considered. Process parameters used in D.I.W pneumatic extrusion based 3D printing have been shown to have influence on electrical conductivity [36-40], dimensional error and surface roughness [41], layer flatness and interfacial strength [42] of the printed circuits. Hagen et al. reported the influence of printing speed, raster angle, flow rate, extrusion temperature on electrical characterization of the circuits printed using carbon black (CB), $\mathrm{CNT}$, copper nanowires and analysed that optimization of raster angle, extrusion temperature play a vital role in printing circuits with highest conductivity [36]. Paz et al. have concluded that increase in layer thickness, nozzle diameter leads to increase in electrical conductivity of 3D printed circuits [37]. Optimization of ink formulations and process printing parameters are studied by Ebers et al. for direct ink writing of cellulose aqueous inks and concluded that adjustment of printing speed, layer thickness effect the shape fidelity and electrical conductivity of the printed circuits [38]. Corral et al. studied the dimensional error effects, surface roughness of printed zirconia parts considering the process variables such as layer height, printing speed, infill, bed temperature and reported that dimensional error depends on the combination of infill rate, layer height where as the surface roughness depends on infill rate and printing speed [41]. Guo et al. have reported that nozzle diameter, strip spacing, printing speed, air pressure play a crucial role in printing circuits with controlled layer flatness, high interfacial strength which are the most important factors in utilization of sensors and concluded that inappropriate strip spacing will create ripples on surface of printed layer [42].

In our previous work [7], strain gage circuit is fabricated for sensing applications by D.I.W method using MWCNT/ PVA composites. However, understanding the variation of electrical conductivity with changes in process parameters is of significance to fabricate a strain gage circuit with high electrical conductivity. Therefore, this work focuses on three crucial parameters viz. nozzle diameter, extrusion pressure, printing speed for fabricating electronic circuits by D.I.W method. Though the present work is associated with previous research findings, the key aspect of this research work is to optimize the process parameters and study its effects on electrical conductivity of 3D printed circuits fabricated by D.I.W method.

This article is organized as: conductive ink preparation, experimental setup, experimental procedure, researched input printing process parameters, design of experiments, circuit printing, electrical conductivity measurement followed by results and discussion.

\section{Materials and methods}

\subsection{Materials used}

Conductive nano filler used in the present work is multiwalled carbon nanotubes, procured from United Nanotech Pvt. Ltd. India. Polyvinyl alcohol with melt flow of $39 \mathrm{~g} / \mathrm{min}$ at $180^{\circ} \mathrm{C}$, molecular weight of $96000 \mathrm{~g} / \mathrm{mol}$ is procured from Merck, India. PVA acts as a binder to develop MWCNT/PVA conductive ink. In order to enhance dispersion of MWCNT in PVA matrix, functionalization of MWCNT is performed using nitric acid and hydrogen peroxide purchased from fisher scientific, India.

\subsection{Preparation F-MWCNT/PVA conductive ink}

$20 \mathrm{~g}$ of PVA gel is prepared by dissolving $3 \mathrm{~g}$ of PVA in $17 \mathrm{~mL}$ of distilled water. Maintaining an $\mathrm{rpm}$ of 500 , temperature $60^{\circ} \mathrm{C}$; diluted PVA was stirred for $60 \mathrm{~min}$ using hot plate process. To this $1.5 \mathrm{wt} . \%$ of F-MWCNT was added manually and mechanically stirred for $2 \mathrm{~h}$ and left for drying at room temperature for a duration of $24 \mathrm{~h}$. Functionalization of MWCNT is initiated with mixing of $0.3 \mathrm{~g}$ of MWCNT in $70 \mathrm{ml}$ of $\mathrm{HNO}_{3}$ and stirred for $20 \mathrm{~min}$ at $50^{\circ} \mathrm{C}$ temperature followed by ultra sonication for $90 \mathrm{~min}$. Obtained solution is dried at $120^{\circ} \mathrm{C}$ in hot oven for $6 \mathrm{~h}$. Attained dry powder is mixed with $\mathrm{H}_{2} \mathrm{O}_{2}$ and stirred for $90 \mathrm{~min}$. Obtained solution is thoroughly washed with water till the $\mathrm{pH}$ of solution is found to be neutral. Here, obtained solution is dried at $50^{\circ} \mathrm{C}$ in hot oven for $12 \mathrm{~h}$ and functionalized MWCNT is obtained.

\subsection{Experimental setup}

Figure 1 presents the schematic of experimental D.I.W process. Conductive inks made from MWCNT and PVA are loaded into syringe barrel attached to head assembly with controlled XY-axis translation. Nozzle of different diameters can be used as per the design pattern. Present work is associated with printing the required prototypes by extrusion of conductive ink using controlled pneumatic pressure. Inks are deposited on to built platform with controlled Z-axis translation. D.I.W 3D printer utilized in the current work to print electronic circuits is indigenously made; supports build volume of $(120 \times 120 \times 120) \mathrm{mm}$ and works on pneumatic extrusion. Actual D.I.W printer used in this work is presented in Figure 2. 


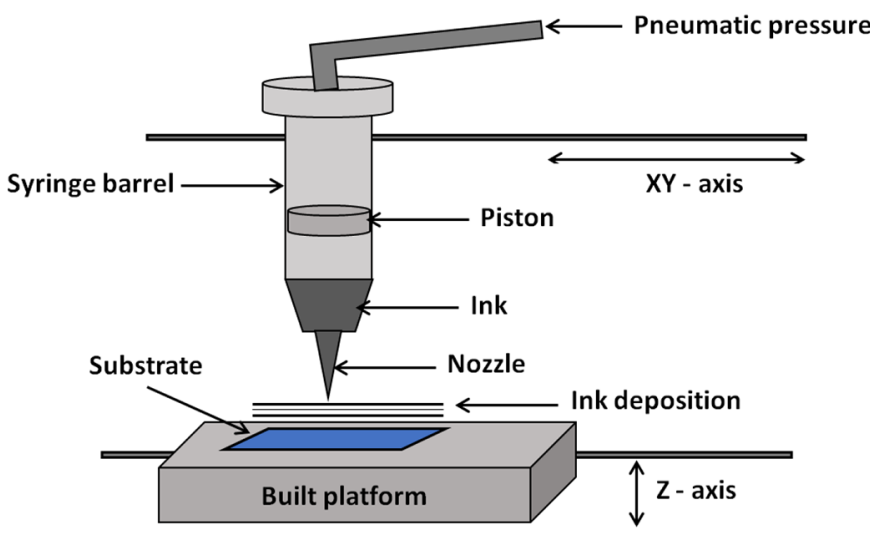

Fig. 1. Graphical illustration of D.I.W process.

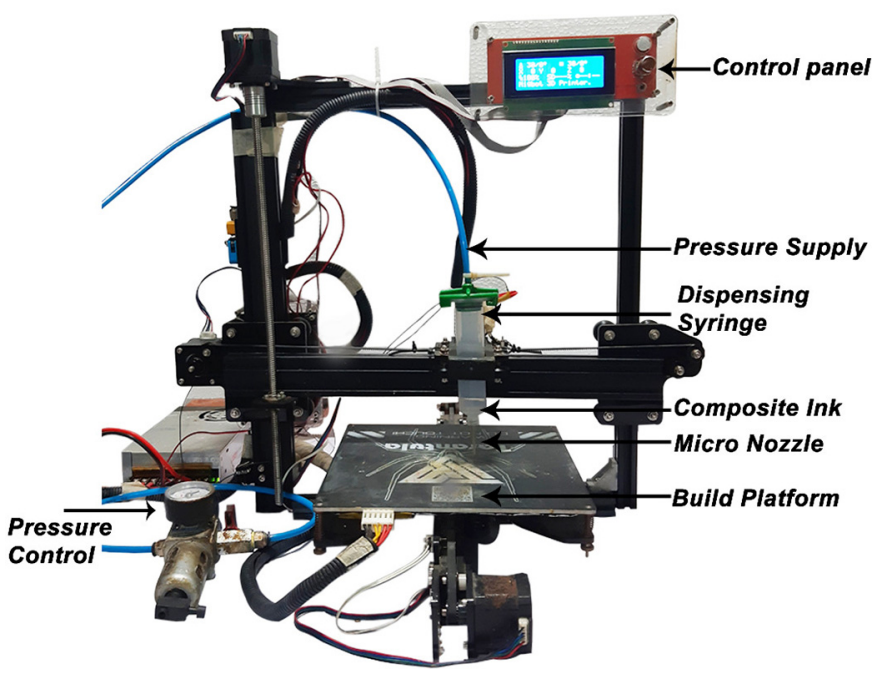

Fig. 2. Actual D.I.W 3D printer utilized in the current work.

Table 1. D.I.W process printing parameters and levels.

\begin{tabular}{llllll}
\hline S. no. & Printing parameters & Units & Level 1 & Level 2 & Level 3 \\
\hline 1 & Nozzle diameter & $\mathrm{mm}$ & 0.6 & 0.8 & 1.0 \\
2 & Extrusion pressure & $\mathrm{MPa}$ & 0.1 & 0.15 & 0.2 \\
3 & Printing speed & $\mathrm{mm} / \mathrm{s}$ & 4 & 7 & 10 \\
\hline
\end{tabular}

\section{Experimental procedure}

\subsection{Selection of printing parameters}

Printing parameters are very essential to enhance the quality and efficiency of the desired part that is to be printed by $3 \mathrm{D}$ printing method. In the present work; nozzle diameter, extrusion pressure, printing speed are selected as input variables and output is observed in terms of electrical conductivity. Input process variables and levels of printing parameters are selected by considering the researched printing process parameters reported from the previous findings [36-39]. Optimization of printing parameters is very essential in the current work to print electronic circuits with high electrical conductivity. Table 1 represents the level of printing parameters used in the present work.

\subsection{Design of experiments using Box-Behnken method}

Box-Behnken method is one of the types of response surface methodology (RSM). This design approach enables to analyse real time problems by reducing the number of experimental trails to be evaluated considering the input parameters and their interaction. Optimization of the response which is influenced by input parameters is the core objective of RSM method. In the present work; considering the cost of materials used, application of RSM will benefit the $3 \mathrm{D}$ printing process reducing the number of experimental trails. RSM based Box-Behnken design is adopted to analyse the influence of input process parameters namely nozzle diameter, extrusion pressure and printing speed on the electrical conductivity of the printed electronic circuits.

\subsection{Optimization of printing parameters and printing of circuits}

D.I.W printer shown in Figure 2 is used to print one layer of circuit lines of length $4 \mathrm{~cm}$ using prepared F-MWCNT/ PVA conductive ink following the different levels of input parameters developed in Table 2. Printed circuit lines are presented in Figure 3a. Electrical conductivity of the printed circuits is calculated using Extech MN-35 multimeter, following the concept of two-point probe measurement method. Varying the input printing process parameters, electrical conductivity observed in the printed lines is presented in Table 2. Figure 3b shows the strain gage circuit printed using the best electrical conductivity observed from input printing parameters as per run number 11 .

\section{Results and discussion}

Significance of the adopted D.I.W 3D printing process has been determined using ANOVA results for electrical conductivity with a confidence level of $95 \%$. In the analysis process; sum of squares, mean square, lack of fit and 
Table 2. Box-Behnken method based experimental design with measured response.

\begin{tabular}{|c|c|c|c|c|}
\hline \multicolumn{5}{|c|}{ Box-Behnken design matrix for 3 level and 3 parameters } \\
\hline Run & $\underline{\text { Level } 1}$ & $\underline{\text { Level } 2}$ & $\underline{\text { Level } 3}$ & $\underline{\text { Response } 1}$ \\
\hline & $\begin{array}{l}\text { A: Nozzle diameter } \\
\mathrm{mm}\end{array}$ & $\begin{array}{l}\mathrm{B}: \text { Extrusion pressure } \\
\mathrm{MPa}\end{array}$ & $\begin{array}{l}\mathrm{C}: \text { Printing speed } \\
\mathrm{mm} / \mathrm{s}\end{array}$ & $\begin{array}{l}\text { Electrical conductivity } \\
\mathrm{S} / \mathrm{m}\end{array}$ \\
\hline 1 & 0.6 & 0.2 & 7 & 711 \\
\hline 2 & 0.6 & 0.15 & 10 & 707 \\
\hline 3 & 0.8 & 0.15 & 7 & 736 \\
\hline 4 & 0.8 & 0.15 & 7 & 735 \\
\hline 5 & 1 & 0.2 & 7 & 688 \\
\hline 6 & 0.8 & 0.2 & 10 & 724 \\
\hline 7 & 0.8 & 0.2 & 4 & 737 \\
\hline 8 & 0.6 & 0.1 & 7 & 717 \\
\hline 9 & 1 & 0.15 & 10 & 684 \\
\hline 10 & 0.8 & 0.15 & 7 & 734 \\
\hline 11 & 0.8 & 0.1 & 4 & 759 \\
\hline 12 & 0.6 & 0.15 & 4 & 723 \\
\hline 13 & 1 & 0.1 & 7 & 705 \\
\hline 14 & 1 & 0.15 & 4 & 712 \\
\hline 15 & 0.8 & 0.1 & 10 & 728 \\
\hline
\end{tabular}

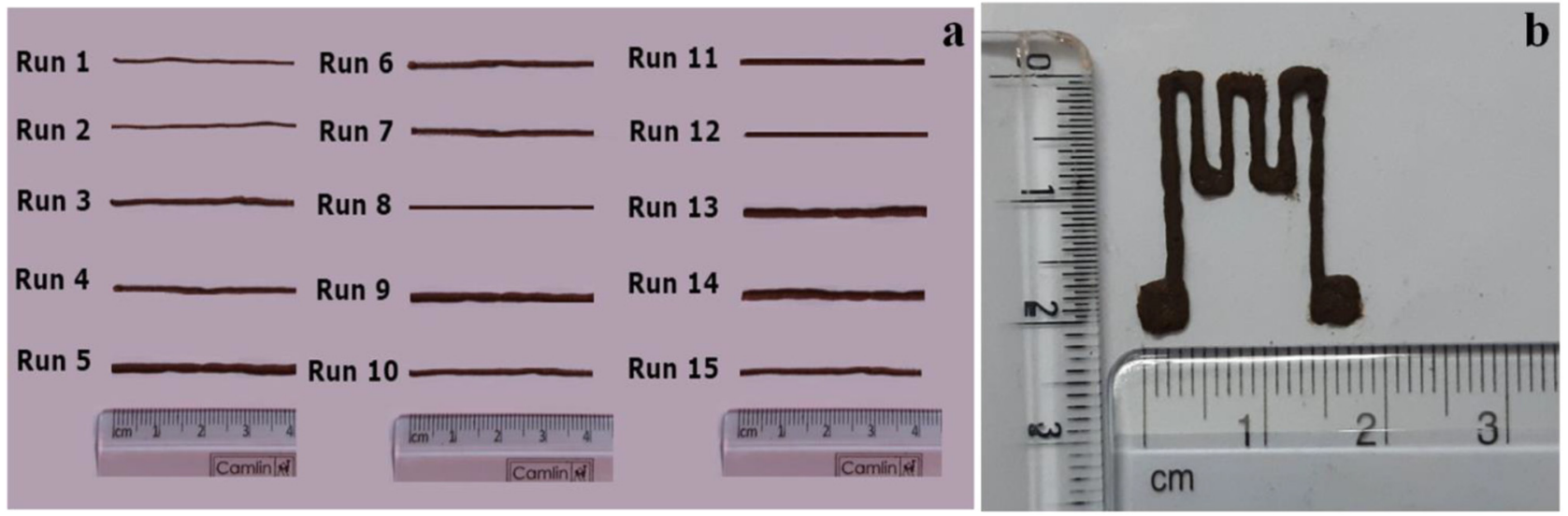

Fig. 3. Circuits printed by D.I.W method (a) 3D printed single layer lines (b) 3D printed strain gage circuit.

F-value with individual interaction for response electrical conductivity is executed. ANOVA is performed to analyse the effects of selected input printing process parameters on electrical conductivity of the $3 \mathrm{D}$ printed circuits.

\subsection{Effect of electrical conductivity and ANOVA}

Influence of printing process parameters on electrical conductivity of the printed circuits is determined using Box-Behnken method. Responses for electrical conductivity are presented in Table 3. Competency of the developed equations is explored by performing significant and lack of fit test. Residuals associated with the developed model is used to create normal $\%$ probability and is plotted in Figure 4; It is observed that all the points fall under a straight line and from this it can be concluded that the assumption model is satisfied by residuals. $F$-value $=604.52$, Prob. $F<0.04$ noted in Table 3 represents that $95 \%$ confidence level is attained in the reported model. Reported value of $R^{2}$ (multiple regression co-efficient) $=0.999403$, Adj. $R^{2}$ (adjusted co-efficient of determination) $=0.998328$ proves that the developed model fits accurately with the actual. Considering the $F$-value of the input printing process parameters, it is found that printing speed and nozzle diameter are the most influencing parameters which affect the electrical conductivity. 
Table 3. ANOVA for electrical conductivity of printed circuits.

\begin{tabular}{llllll}
\hline Source variance & Sum of squares & DoF & Mean square & F-value & $P$-Value \\
\hline Model & 5440.75 & 9 & 604.52778 & 930.042735 & $<0.0001$ \\
A-Nozzle diameter & 595.125 & 1 & 595.125 & 915.5769231 & $<0.0001$ \\
B-Extrusion pressure & 300.125 & 1 & 300.125 & 461.7307692 & $<0.0001$ \\
C-Printing speed & 968 & 1 & 968 & 1489.230769 & $<0.0001$ \\
$\mathrm{AB}$ & 30.25 & 1 & 30.25 & 46.53846154 & 0.0010 \\
$\mathrm{AC}$ & 36 & 1 & 36 & 55.38461538 & 0.0007 \\
$\mathrm{BC}$ & 81 & 1 & 81 & 124.6153846 & 0.0001 \\
$\mathrm{~A}^{2}$ & 3350.8269 & 1 & 3350.8269 & 5155.118343 & $<0.0001$ \\
$\mathrm{~B}^{2}$ & 0.5192308 & 1 & 0.5192308 & 0.798816568 & 0.4124 \\
$\mathrm{C}^{2}$ & 9.75 & 1 & 9.75 & 15 & 0.0117 \\
Residual & 3.25 & 5 & 0.65 & & \\
Lack of fit & 1.25 & 3 & 0.4166667 & 0.416666667 & 0.7615 \\
Pure error & 2 & 2 & 1 & & \\
Cor. total & 5444 & 14 & & & \\
$R^{2}$ & 0.999403 & Adj. $R^{2}$ & 0.998328 & & \\
\hline
\end{tabular}

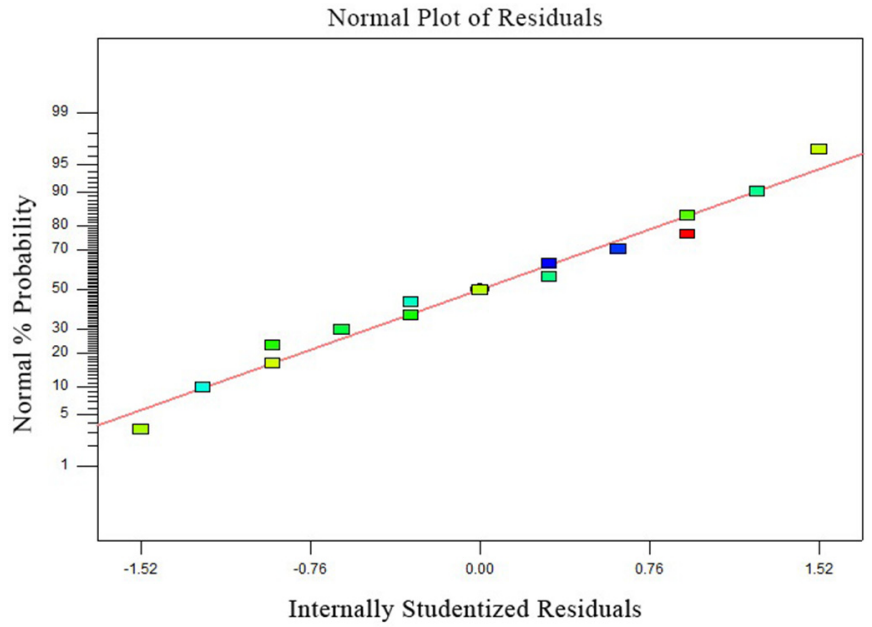

Fig. 4. Normal plot of residuals.

Relation between input printing process parameters and electrical conductivity is attained using equation (1).

See equation (1) below.

where, $\mathrm{A}=$ Nozzle diameter, $\mathrm{B}=$ Extrusion pressure, $\mathrm{C}=$ Printing speed. Equation (1) gives the optimization value of all the input process parameters with the high electrical conductivity value.

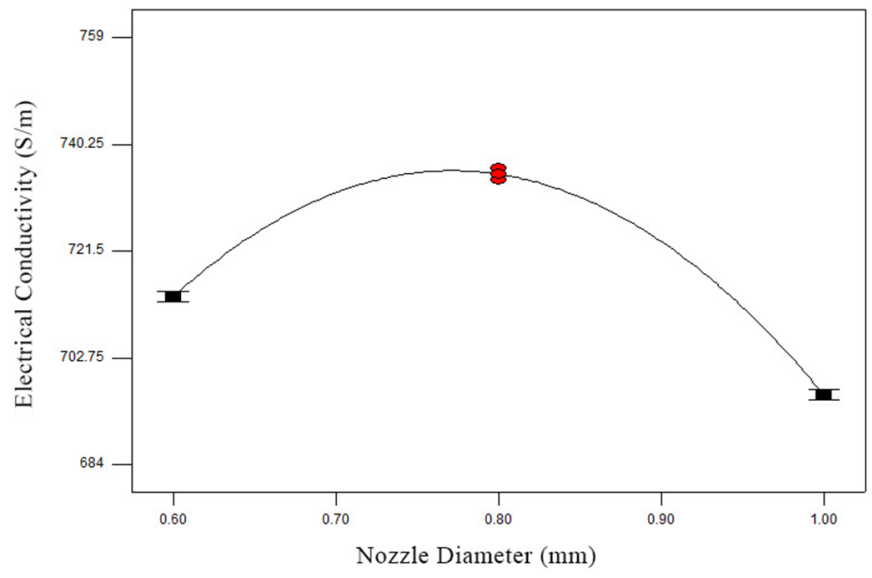

Fig. 5. Electrical conductivity with varying nozzle diameter.

\subsection{Printing parameters influence on electrical conductivity}

Electrical conductivity is one of the most important characterizations in direct ink writing of electronic circuits that influences the performance of a circuit in sensing applications. Higher electrical conductivity is preferred in sensors to achieve high performance. Results reported with variation of nozzle diameter, presented in Figure 5 showed that $0.8 \mathrm{~mm}$ of nozzle diameter would be optimum to print circuit. Increasing the diameter of nozzle by $0.2 \mathrm{~mm}$

Electrical Conductivity $=314.2638889+1238.125 \times \mathrm{A}-157.5 \times \mathrm{B}-6.694444444$

$$
\begin{aligned}
& \times \mathrm{C}-275 \times \mathrm{A} \times \mathrm{B}-5 \times \mathrm{A} \times \mathrm{C}+30 \times \mathrm{B} \times \mathrm{C}-753.125 \times \mathrm{A}^{2} \\
& +150 \times \mathrm{B}^{2}+0.180555556 \times \mathrm{C}^{2}
\end{aligned}
$$




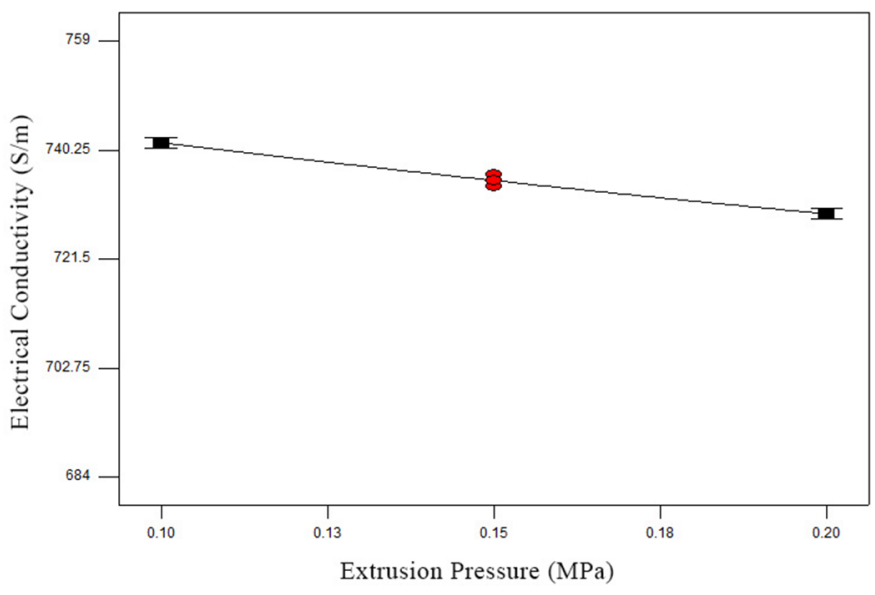

Fig. 6. Electrical conductivity with varying extrusion pressure.

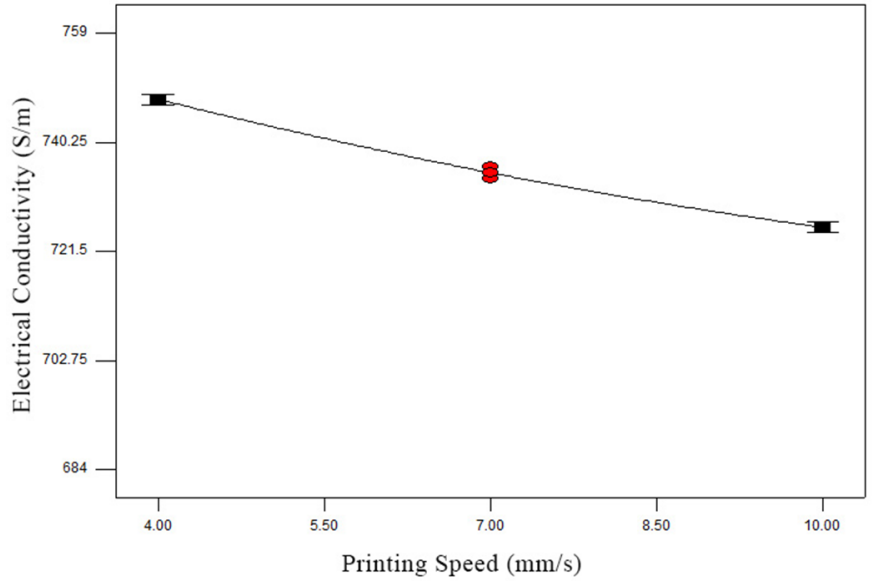

Fig. 7. Electrical conductivity with varying printing speed.

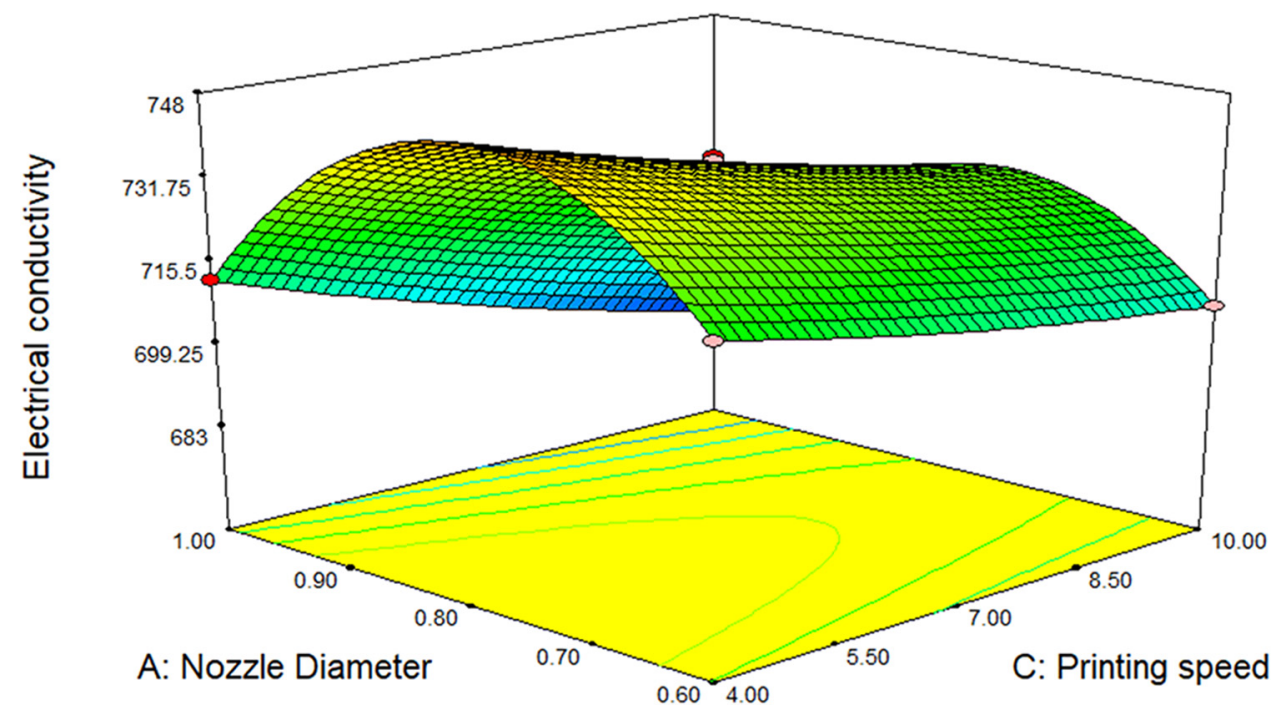

Fig. 8. Effects of nozzle diameter and printing speed on electrical conductivity.

reduces the conductivity. Figure 6 presents the reported electrical conductivity values with the variation of extrusion pressure. It is observed that with increase in extrusion pressure, conductivity decreases. Variation of printing speed and its impact on electrical conductivity is shown in Figure 7 and it is noted that increase in printing speed has contributed to high $\%$ in reduction of conductivity.

It is observed from Table 3 that printing speed, nozzle diameter are the most significant input printing process variables that influence electrical conductivity and effect of extrusion pressure is substandard. Input process variables effect on electrical conductivity is presented in Figures 8-10. The effects of nozzle diameter, printing speed on electrical conductivity illustrated in Figure 8 designates that electrical conductivity increases with reduction in nozzle diameter, printing speed. With the usage of bigger nozzle and higher printing speed, ink will be deposited without uniformity and leads to decrease in electrical conductivity. From Figure 9, changes in electrical conductivity with the variation of extrusion pressure, nozzle diameter are analysed and noted that electrical conductivity decreases with increase in extrusion pressure, nozzle diameter. This may be due to the deposition of more ink than required to print a desired circuit leading to the formation of elevated or rough surface circuits with low conductivity. Figure 10 presents the effects of electrical conductivity on the variation of printing speed and extrusion pressure where electrical conductivity decreases with increase in printing speed and extrusion pressure. Analysing the changes in electrical conductivity with variable input printing process parameters it is observed that adequate printing speed and nozzle diameter are very significant to print circuits with high electrical conductivity. 


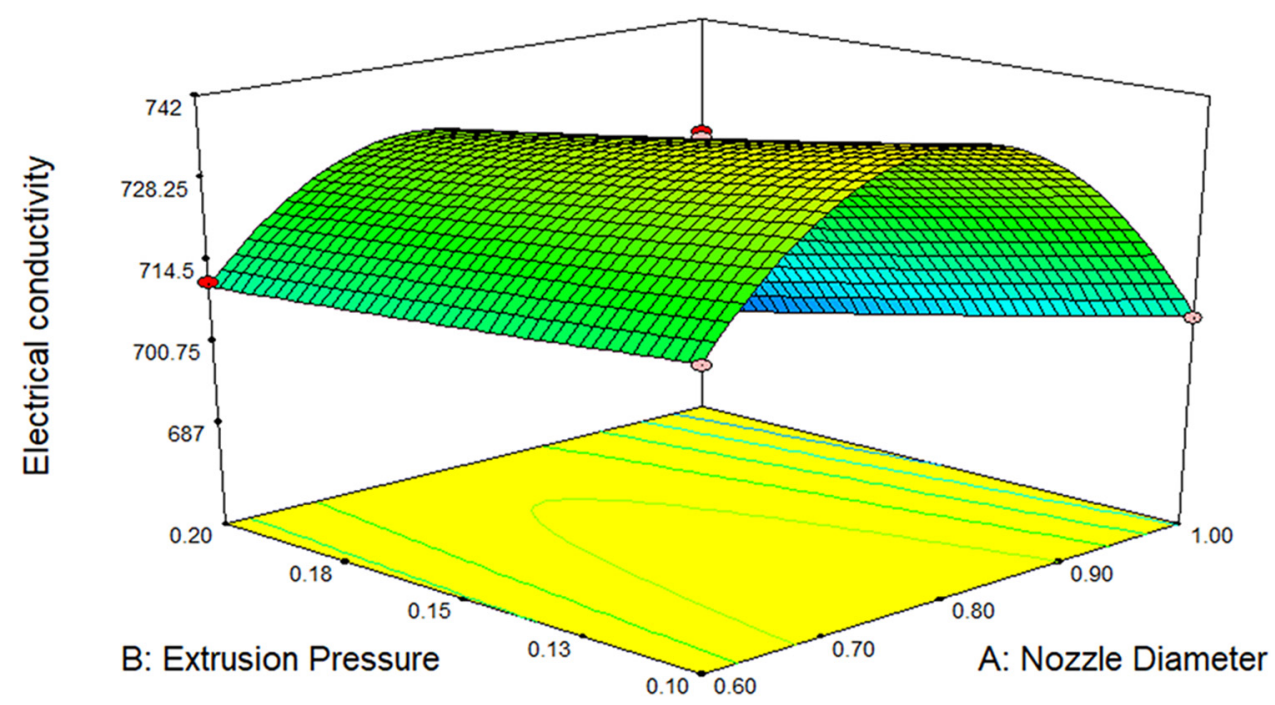

Fig. 9. Effects of nozzle diameter and extrusion pressure on electrical conductivity.

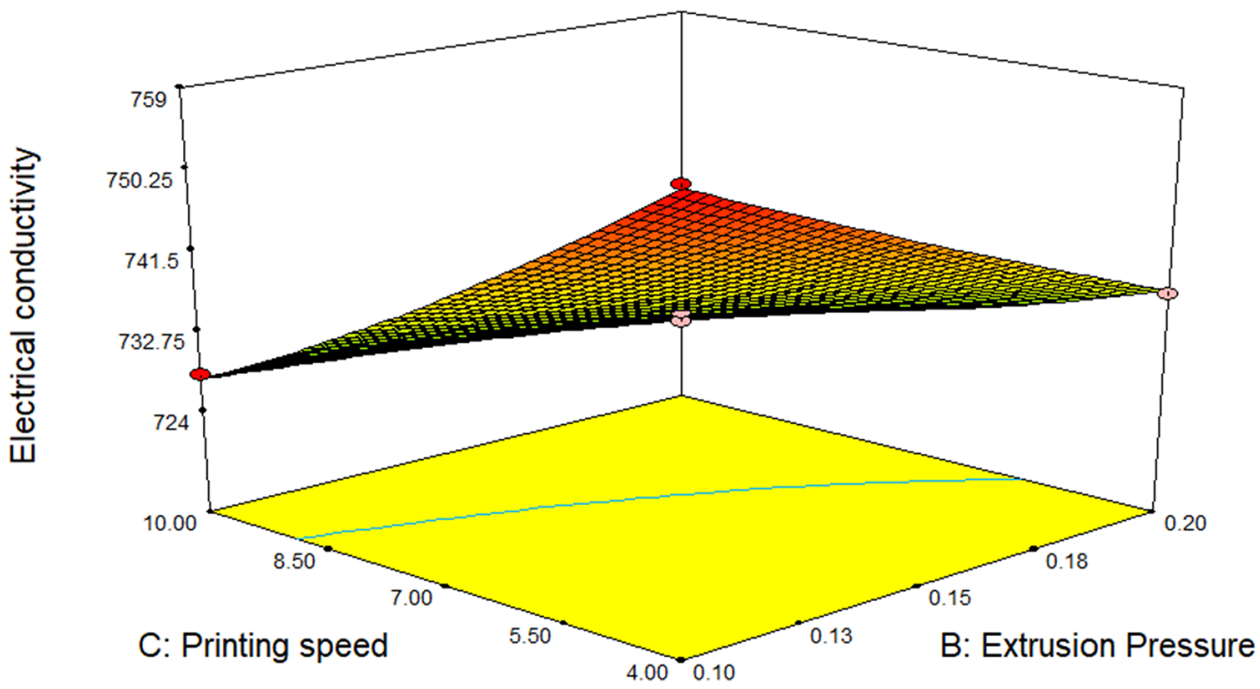

Fig. 10. Effects of extrusion pressure and printing speed on electrical conductivity.

\section{Conclusion}

- This work represents the influence of three input printing process parameters (nozzle diameter, extrusion temperature, and printing speed) on the electrical conductivity response of the circuits printed by D.I.W method.

- In this study, it was found that nozzle diameter and printing speed are the most significant input printing process variables that influence electrical conductivity. With increase in nozzle diameter and printing speed, electrical conductivity decreases. This may be attributed due to the deposition of more ink than required to print a desired circuit leading to the formation of elevated or rough surface circuits with low conductivity.

- Optimized printing parameters such as nozzle diameter of $0.8 \mathrm{~mm}$, extrusion pressure of $0.1 \mathrm{MPa}$ and printing speed of $4 \mathrm{~mm} / \mathrm{s}$ are found to be ideal for printing electronic circuits with high electrical conductivity.

\section{References}

1. P. Jiang et al., Recent advances in direct ink writing of electronic components and functional devices, Prog. Addit. Manuf. 3, 65-86 (2018)

2. A.H. Espera et al., 3D-printing and advanced manufacturing for electronics. Prog. Addit. Manuf. 4, 245-267 (2019)

3. Y. Zhang et al., Recent progress of direct ink writing of electronic components for advanced wearable devices, ACS Appl. Electron. Mater. 1, 1718-1734 (2019)

4. M. Abas et al., Direct ink writing of flexible electronic circuits and their characterization, J. Braz. Soc. Mech. Sci. Eng. 41, 1-11 (2019)

5. Y. Liu et al., Handwriting flexible electronics: tools, materials and emerging applications, J. Sci.: Adv. Mater. Devices 5, 451-467 (2020)

6. Y. Chen et al., Direct ink writing of flexible electronics on paper substrate with graphene/polypyrrole/carbon black ink, J. Electron. Mater. 48, 3157-3168 (2019) 
7. S.R. Ahammed, A.S. Praveen, Direct ink writing method for manufacturing electronic circuits using multiwalled carbon nanotubes and polyvinyl alcohol composites, Mater. Perform. Charact. 9, 665-674 (2020)

8. B. Luo et al., Printing carbon nanotube-embedded silicone elastomers via direct writing, ACS Appl. Mater. Interfaces 10, 44796-44802 (2018)

9. S. Chen et al., A single integrated 3D-printing process customizes elastic and sustainable triboelectric nanogenerators for wearable electronics, Adv. Funct. Mater. 28, 1805108 (2018)

10. M.M. Ovhal, N. Kumar, J.-W. Kang, 3D direct ink writing fabrication of high-performance all-solid-state micro-supercapacitors, Mol. Cryst. Liquid Cryst. 705, 105-111 (2020)

11. B. Chen et al., Fully packaged carbon nanotube supercapacitors by direct ink writing on flexible substrates, ACS Appl. Mater. Interfaces 9, 28433-28440 (2017)

12. J. Zhao et al., Direct ink writing of adjustable electrochemical energy storage device with high gravimetric energy densities, Adv. Funct. Mater. 29, 1900809 (2019)

13. M. Wei et al., 3D direct writing fabrication of electrodes for electrochemical storage devices, J. Power Sources 354, 134-147 (2017)

14. G. Shi et al., A versatile PDMS submicrobead/graphene oxide nanocomposite ink for the direct ink writing of wearable micron-scale tactile sensors, Appl. Mater. Today 16, 482-492 (2019)

15. Z. Wang et al., 3D printed graphene/polydimethylsiloxane composite for stretchable strain sensor with tunable sensitivity, Nanotechnology 30, 345501 (2019)

16. Y. Hu et al., A low-cost, printable, and stretchable strain sensor based on highly conductive elastic composites with tunable sensitivity for human motion monitoring, Nano Res. 11, 1938-1955 (2018)

17. H. Li et al., 3D printed flexible triboelectric nanogenerator with viscoelastic inks for mechanical energy harvesting, Nano Energy 58, 447-454 (2019)

18. N.M. Nair et al., Direct writing of silver nanowire-based ink for flexible transparent capacitive touch pad, Flexible Printed Electron. 4, 045001 (2019)

19. K. Shrivas et al., The direct-writing of low cost paper based flexible electrodes and touch pad devices using silver nanoink and ZnO nanoparticles, RSC Adv. 9, 17868-17876 (2019)

20. O. Gubanova et al., Fabrication and package of ISFET biosensor for micro volume analysis with the use of direct ink writing approach, Mater. Sci. Semicond. Process. 60, 71-78 (2017)

21. J. Chen et al., Highly stretchable photonic crystal hydrogels for a sensitive mechanochromic sensor and direct ink writing, Chem. Mater. 31, 8918-8926 (2019)

22. H.A. Loh et al., Direct ink writing of graphene-based solutions for gas sensing, ACS Appl. Nano Mater. 2, 4104-4112 (2019)

23. M. Ma, H. Zhang, An experimental study of pneumatic extruding direct writing deposition-based additive manufacturing, Int. J. Adv. Manuf. Technol. 97, 1005-1010 (2018).

24. J.K. Placone, A.J. Engler, Recent advances in extrusionbased 3D printing for biomedical applications, Adv. Healthcare Mater. 7, 1701161 (2018)
25. K.-W. Chen, M.-J. Tsai, H.-S. Lee, Multi-nozzle pneumatic extrusion-based additive manufacturing system for printing sensing pads, Inventions 5, 29 (2020)

26. J. Zhao et al., Printable ink design towards customizable miniaturized energy storage devices, ACS Mater. Lett. 2, 1041-1056 (2020)

27. S. Schlisske et al., Ink formulation for printed organic electronics: investigating effects of aggregation on structure and rheology of functional inks based on conjugated polymers in mixed solvents, Adv. Mater. Technol. 6, 2000335 (2021)

28. C. O'Mahony et al., Rheological issues in carbon-based inks for additive manufacturing, Micromachines 10, 99 (2019)

29. C. Xu et al., Direct ink writing of DNTF based composite with high performance, Propellants Explos. Pyrotechn. 43, 754-758 (2018)

30. V.G. Rocha et al., Direct ink writing advances in multimaterial structures for a sustainable future, J. Mater. Chem. A 8, 15646-15657 (2020)

31. S. Nesaei et al., Additive manufacturing with conductive, viscoelastic polymer composites: direct-ink-writing of electrolytic and anodic poly (ethylene oxide) composites, J. Manuf. Sci. Eng. 139 (2017)

32. A.A. Farizhandi et al., Synthesized biocompatible and conductive ink for 3D printing of flexible electronics, J. Mech. Behav. Biomed. Mater. 110, 103960 (2020)

33. A. Koutsioukis et al., Highly conductive water-based polymer/graphene nanocomposites for printed electronics, Chem. - Eur. J. 23, 8268-8274 (2017)

34. Y. Jiang et al., Direct ink writing of wearable thermoresponsive supercapacitors with $\mathrm{rGO} / \mathrm{CNT}$ composite electrodes, Adv. Mater. Technol. 4, 1900691 (2019)

35. Y. Wang et al., Direct graphene-carbon nanotube composite ink writing all-solid-state flexible microsupercapacitors with high areal energy density, Adv. Funct. Mater. 30, 1907284 (2020)

36. H. Watschke, K. Hilbig, T. Vietor, Design and characterization of electrically conductive structures additively manufactured by material extrusion, Appl. Sci. 9, 779 (2019)

37. R. Paz et al., Influence of manufacturing parameters and post processing on the electrical conductivity of extrusion-based 3D printed nanocomposite parts, Polymers 12, 733 (2020)

38. L.-S. Ebers, M.-P. Laborie, Direct ink writing of fully biobased liquid crystalline lignin/hydroxypropyl cellulose aqueous inks: optimization of formulations and printing parameters, ACS Appl. Bio Mater. 3, 6897-6907 (2020)

39. D. Rajamani et al., Experimental investigations and parametric optimization of process parameters on shrinkage characteristics of selective inhibition sintered high density polyethylene parts, Exper. Techn. 42, 631-644 (2018)

40. K. Ananthakumar et al., Measurement and optimization of multiresponse characteristics in plasma arc cutting of Monel $400^{\mathrm{TM}}$ using RSM and TOPSIS, Measurement 135, 725-737 (2019)

41. I. Buj-Corral et al., Effect of printing parameters on dimensional error and surface roughness obtained in direct ink writing (DIW) processes, Materials 13, 2157 (2020)

42. Z. Guo et al., High-precision resistance strain sensors of multilayer composite structure via direct ink writing: optimized layer flatness and interfacial strength, Compos. Sci. Technol. 201, 108530 (2021)

Cite this article as: Syed Riyaz Ahammed, Ayyappan Susila Praveen, Optimization parameters effects on electrical conductivity of 3D printed circuits fabricated by direct ink writing method using functionalized multiwalled carbon nanotubes and polyvinyl alcohol conductive ink, Int. J. Simul. Multidisci. Des. Optim. 12, 7 (2021) 